This is the peer reviewed version of the following article: Maslin K, Dean $T$, Arshad SH, Venter C. Fussy eating and feeding difficulties in infants and toddlers consuming a cows' milk exclusion diet. Pediatr Allergy Immunol 2015: 26: 503-508, which has been published in final form at http://onlinelibrary.wiley.com/doi/10.1111/pai.12427/full. This article may be used for non-commercial purposes in accordance with Wiley Terms and Conditions for Self-Archiving.

\title{
Fussy eating and feeding difficulties in infants and toddlers consuming a cows' milk exclusion diet
}

\section{Introduction}

2 Cows' Milk Allergy (CMA) is known to affect $\sim 3 \%$ of children in the UK (1). It is also known that parents may incorrectly perceive their child to have a food allergy (2) and that allergen avoidance diets are sometimes initiated unnecessarily $(3,4)$. In practice this means that many children are excluding a major food group from their diet at a time in life that is critical for growth, development and establishment of eating habits. Infants with CMA who are not breastfed are prescribed hypoallergenic infant formulae, which have an altered taste. Parents are also advised that their child should follow a special weaning diet avoiding all forms of cows' milk, usually until at least one year of age, but this exclusion diet may continue for much longer.

Fussy eating and feeding difficulties are separate entities, that may co exist. Fussy eating, generally defined as "consuming a limited variety of food" is a very common problem in young children (5). Up to $20 \%$ of infants and toddlers in the UK are reported to be "problem" eaters by their parents (6) with some studies reporting up to $50 \%$ are fussy eaters (7). In healthy infants and toddlers, it is known that development of feeding skills occurs from 0-24 months with individual variation in gaining self-feeding fine motor skills (8). Feeding difficulties refers to a spectrum of problematic eating behaviours such as excessive spitting out of food, crying/irritability at feeding time, eating extremely slowly, retching at the sight of bottle or spoon, apparent difficulty in swallowing, throwing and pushing away food (Crist \& NapierPhillips, 2001; Lewinsohn et al., 2005). Feeding difficulties are known to be more common in certain medical conditions (e.g. autism spectrum disorder) (11).

In a young child with suspected or confirmed food allergy, where at least one food group is already being restricted, fussy eating and feeding difficulties are likely to have a considerable impact on eating habits and food intake. To date there has been limited research directly investigating the prevalence of these eating problems in children consuming a special diet for food allergy (12). The existing studies have 
mainly recruited children with severe non-IgE mediated gastrointestinal disease and have not included a control group of children eating a normal diet $(13,14)$. The aim of this study is to determine the prevalence of fussy eating and feeding difficulties in infants and toddlers consuming a Cows' Milk Exclusion (CME) diet compared to a control group consuming an unrestricted diet. If found to be more prevalent, intervention by a qualified dietitian will ensure timely diagnoses and appropriate advice to prevent long-term consequences of fussy eating habits.

\section{Methods}

\section{Study design}

This was a cross sectional study of 8-30 month old children from the Isle of Wight, United Kingdom. This study included two groups: an experimental group, composed of children consuming a CME diet for the treatment of presumed CMA and a control group of children consuming an unrestricted diet. Children were eligible for inclusion in the experimental group if they had consumed a hypoallergenic formula and/or a CME diet in the first year of life for a period of 3 months or longer and or if they were excluding other foods (e.g. egg or soya).

Recruitment took place between July 2013 and December 2014. Participants eligible for the experimental group were identified via routine allergy clinics. The control group was recruited from health visitor clinics in the same locality. Ethical approval was obtained from Berkshire NHS ethics committee.

\section{Data collection}

Fussy eating and feeding difficulties were measured using two separate questionnaires. Fussy eating was measured using the Picky Eater questionnaire (15). It consists of 10 items describing specific behaviours related to fussy eating with questions such as "overall to what extent does your child like a wide variety of foods from those that you think he/she should eat?" and "how often do you prepare a special food for your child because he/she does not like what the rest of the family is eating?". Feeding difficulties was measured using the Montreal Children's Hospital Feeding Difficulties questionnaire (16). It consists of 14 comprehensive questions, covering the following feeding domains: oral motor, oral sensory, appetite, maternal concerns about feeding, mealtime behaviours, maternal strategies used and family reactions to child's feeding. Information was also collected on social demographics, family history of allergy, allergic symptoms, infant feeding and growth. 
67 A power calculation for a two-tailed outcome, at $80 \%$ power indicated that 124 participants were required in this study. Questionnaires were scored and coded according to published guidelines. Data was analysed using SPSS software (IBM, version 20). Descriptive statistics were calculated. Differences between the CME and control groups were compared using Mann Whitney or $\mathrm{X}^{2}$ test. Spearmann rho correlations were performed. Multiple regression calculations were performed to determine the contributing factors to the main outcome variables. A significance level of $p<0.05$ was set for all analyses.

\section{Results}

\section{Description of sample}

126 participants were recruited. Demographic characteristics are detailed in Table 1. Participants in the CME group were younger than those in the control group $(p=$ 0.02 ), but the age range was the same. There were no differences in gender, number of siblings, ethnicity, maternal age/education or growth measurements between the two groups.

83

\section{Infant feeding and dietary exclusion}

Details of participants' infant feeding history are shown in Table 2. The majority of infants had been breastfed at some stage (81\%), but only $13.5 \%$ were being breastfed at the time of data collection. Infants in the control group were commenced on solid food ( $p=0.033)$, lumpy food $(p=0.049)$ and finger foods $(p=0.000)$ significantly earlier than the CME group.

$71.2 \%$ of the CME group was excluding cows' milk only, whilst $28.8 \%$ were excluding another food allergen in addition to cows' milk. Cows' milk was excluded at a median age of 9.5 weeks (range 1-30). Three infants in the CME group were breastfed as their main source of milk and did not have any substitute formula. At the time of data collection, the median duration of a hypoallergenic formula use was 41.0 weeks (range 2-91 weeks). The most commonly used hypoallergenic formula was Amino Acid Formula (45.5\%), followed by Extensively Hydrolysed $(E H)$ whey formula $(25.8 \%)$ and $\mathrm{EH}$ casein formula (16.6\%).

\section{Reported symptoms and SPT status}

Participants in the CME group reported a median number of 4.0 symptoms (ranging from 1-7 symptoms). Participants whose mother had a history of food allergy had 
significantly more symptoms reported $(p=0.000)$, with reported higher rates of vomiting $(p=0.037)$, abdominal pain $(p=0.000)$ and colic $(p=0.004)$ than those with no maternal history of food allergy. Twenty participants $(30.3 \%)$ in the CME group had a positive SPT to cows' milk ( $>3 \mathrm{~mm}$ ). Participants who had a positive SPT to cows' milk reported significantly more symptoms $(p=0.006)$.

\section{Main outcome measures}

\section{Feeding difficulties}

The median feeding difficulty score in the CME group (26.5, range 16-68) was significantly higher than that of the control group $(22.0$, range 15-53) $(p<0.01)$, although both groups were within the normal range $(<45)$. Nine participants in the CME group (13.6\%) had scores diagnostic of clinical feeding difficulties (> 45), compared to only one participant in the control group (1.6\%). There was no affect of gender, being older or younger than 12 months, or breastfeeding status on feeding difficulty score. Participants whose mothers had a history of food allergy symptoms recorded significantly higher scores of feeding difficulties $(p=0.03)$.

Within the CME group, there was no correlation between feeding difficulty score and age at introduction of hypoallergenic formula, duration or type of hypoallergenic formula consumption or SPT status. However, some symptoms were found to be significantly correlated with a higher feeding difficulty score. These are listed in Table 3. In addition, the amount of milk substitute formula consumed per day and "attention paid to healthy eating" were also found to be significantly correlated to a higher feeding difficulty score as was a younger age at time of initiating the exclusion diet. Maternal age, age of child, parental education, number of siblings, duration of breastfeeding, age of introduction of solid/lumpy food and duration of exclusion diet were not correlated with feeding difficulty score.

A standard entry multiple regression analysis was undertaken on the CME group to determine the ability of several factors to predict the level of feeding difficulties. In the final model, $41.3 \%$ of the variance in feeding difficulties could be explained $(R=0.642$, SE 11.09). A history of colic made the most contribution to this model $(B$ score $=-0.459, p=0.03$ ). Three variables made a unique statistically significant contribution (colic, dry cough at night and other food related problems). Details are shown in Table 4.

\section{Fussy Eating}


The CME group had a significantly higher median score (22.5, range 10-63) than the control group (18.0, range 10-44) $(p<0.01)$, indicating they have higher levels of fussy eating, although both groups' median scores could be considered in the nonfussy range(15). Overall there was no difference in scores for gender, being older or younger than 12 months, maternal food allergy history or breastfeeding status. Within the CME group, there was no correlation between fussy eating score and age at introduction of hypoallergenic formula, duration of hypoallergenic formula consumption, type of hypoallergenic formula or SPT status. A positive correlation existed for volume of milk substitute consumed per day (Table 3).

\section{Discussion}

This study set out to compare level of feeding difficulties and fussy eating in two groups of young children; one group consuming a CME diet for CMA and a control group consuming an unrestricted diet. Overall we demonstrated that the CME group scored significantly higher for fussy eating and feeding difficulties, although the results for both groups were within normal ranges. Feeding difficulties were found to be significantly positively correlated with a number of allergic symptoms and both variables were found to be correlated with a higher volume of milk substitute consumed per day.

The higher scores observed on the feeding difficulty questionnaire in the CME group was statistically significant. This is the first time this has been reported in a study of infants with suspected CMA using a control group and a validated questionnaire. However it should not be overlooked that both groups had median scores well within normal levels. Indeed the number of children in the control group with feeding difficulties (1.6\%) is considerably lower than that reported in previous studies of normal healthy developing children $(6,9)$, however the methodology for those studies was different.

Studies of feeding difficulties and food allergy have typically been conducted on children with complex gastrointestinal allergies $(13,14,17)$, or in children who also have an underlying comorbidity (18), therefore the participants are not necessarily reflective of the "typical" infant with CMA. Meyer et al. $(n=437)$ found that $30-40 \%$ of children with Food Protein-Induced Gastrointestinal Allergies (FPIGA) had feeding difficulties reported in their medical notes, with a higher rate in those with symptoms of abdominal pain, vomiting, bloating and constipation. Although there are differences between that study and this; there are some commonalities. They 
identified a significant correlation between feeding difficulties and extra-intestinal manifestations (joint pain, lethargy, headaches). Likewise this study identified a significant correlation between non-gastrointestinal allergic symptoms (wheeze and cough) and feeding difficulty score, illustrating that childhood eating/feeding habits are influenced by a wide range of health-related factors. It is known that oral eating requires the coordination of a suck-swallow-breathe pattern and it may be that difficulties in sensory processing are related to cardiorespiratory symptoms including those present in asthma (19). Feeding difficulties are also reported in children with other respiratory conditions $(20,21)$.

Similar to the study by Crist et al (9), feeding difficulty score was not found to be related to socioeconomic status or birth order/number of siblings. Contrary to previous studies $(22,23)$, a link between the age of introduction of any type of solid foods and feeding difficulty score was not identified. Introduction of lumpy foods did contribute to the multiple regression model predicting higher feeding difficulty score, however only in combination with other variables. However, it must be highlighted that the reporting of age of introduction of solid food was based on parent recall, which may affect the accuracy of this data.

Overall infants in the CME group scored significantly higher on their fussy eating questionnaire than the control group. However the median score of 22.5 , is still well below the maximum questionnaire score of 70 , indicating that as a whole the group were not particularly fussy eaters. In a previous study of 2-3 year old children, "picky eaters" were found to have a mean score of 34.3 , compared to "non-picky eaters" who had a mean score of 22.7(15). A study of 12 month old infants examining the role of food texture and fussiness reported a mean score of 25 on a subscale of the questionnaire (24), which is similar to our findings.

No correlations were identified between fussy eating and allergic symptoms. A recent study of 4 year old children in Holland identified a bidirectional correlation between constipation and fussy eating (25). They found no difference in fussy eating levels between those with and without CMA history (personal communication Tharner, January 2015). Other studies have reported that fussy eating occurs across different socioeconomic statuses, genders, ethnic groups and ages (15), which is consistent with our findings. Across all participants, no difference in fussy eating score was found in relation to maternal age or education/occupation status. It is notable that the total volume of milk/milk substitute consumed/day was positively correlated with fussy eating score. This supports the simple dietetic advice to reduce excessive consumption of formula in order to encourage a better appetite and mealtime behaviour. 
Fussy eating can be difficult to quantify accurately and is usually evaluated by

212 a parental report tool or asking of a single yes/no question, rather than analysis of 213 dietary records (26). Although several tools have been developed for measurement 214 of preschool children's fussy eating behaviour, none have been specifically designed 215 for children under 18 months old and this was identified as a gap in the literature in a 216 recent review (27). The questionnaire used in this study was chosen as it has been 217 validated against behavioural measures of eating in 12-month old infants (24) and 218 against two types of dietary records in children aged 24-36 months old.

219 The measurement of feeding difficulties can also be problematic due to the 220 variability in definitions used. In many cases feeding difficulties are transient; 221 however it is not always straightforward to distinguish feeding problems that are likely 222 to be short-lived from those that are more persistent (28). By comparison, the term 223 "Infant Feeding Disorder" is a formal diagnosis used in the current diagnostic 224 systems of the World Health Organisation ICD-10 (29) and Diagnostic and Statistical 225 Manual of Mental Disorders, $4^{\text {th }}$ Edition (30). Both sets of criteria specify that an 226 infant feeding disorder is a persistent failure to eat adequately, associated with 227 weight loss/ significant failure to gain weight, that is not directly due to a medical 228 condition or another mental disorder, with onset before 6 years of age. As many 229 children who consume exclusion diets maintain a normal weight and have an 230 underlying disorder (i.e. food allergy), the use of this definition was not appropriate 231 for this study. Other classification systems such as the Chatoor criteria and Wolfson 232 criteria (31) have been developed, but both involve lengthy questionnaires. The 233 Montreal Hospital Children's feeding scale questionnaire is, to the authors' 234 knowledge, the only validated questionnaire for measurement of feeding difficulties in 235 children under two years of age (16). It is an easy to use measurement that has been 236 demonstrated to be valid and reliable in children with and without medical diagnoses 237 and could be quickly administered in an outpatient setting, in approximately five 238 minutes, with good reliability and internal consistency.

\section{Limitations and strengths of study}

241 There are some limitations to this study. There may be a recruitment bias whereby 242 those more interested in diet are more likely to participate. The method used is 243 reliant on subjective parental report. Parental feeding behaviours, which have the 244 potential to influence infant feeding behaviours (32) were not assessed. The control 245 group was slightly older than CME group, which may have skewed the results 246 slightly. The CME group included participants consuming both single and multiple 
247 exclusion diets. As this was a typical caseload of patients from a secondary care

248 allergy clinic, participants were diagnosed with CMA using clinical history, SPT and

249 dietary exclusion/reintroduction, rather than an oral food challenge. As correlations

250 are reported, causality cannot be confirmed.

251 The strengths of this study are the use of a control group, which was recruited 252 from the same geographical locality as the CME group. The groups were closely 253 matched for all demographic variables; only participant age differed by three months.

254 As the research took place in a secondary care allergy clinic, the results are broadly 255 generalisable to the majority of other clinics around the UK. The fact that the infant 256 feeding data of the group as a whole is so similar to national feeding trends 257 demonstrates that the control group is also reflective of the general population. The 258 recruitment target of the study was met, meaning the study was sufficiently powered. 259 Validated and age-specific questionnaires were used. Data collection, coding, 260 analysis and interpretation took place by the same researcher to minimise the effect 261 of researcher bias.

262

\section{Conclusion}

264

265 In summary, it has been demonstrated that infants consuming a CME diet for CMA 266 have significantly higher scores of feeding difficulties and fussy eating than a control 267 group consuming an unrestricted diet. This may be due to the underlying disease 268 process resulting in allergic symptoms, the restrictive nature of the CME diet or due 269 to feeding practices adapted by the parent and child. The number of allergic 270 symptoms was the factor that was most strongly correlated with feeding difficulties, 271 however type of symptoms was also important, as was the volume of milk substitute 272 consumed per day. However, it should be emphasised that the feeding difficulties 273 and fussy eating scores across the whole group were within normal ranges and there 274 was no effect seen on growth. This provides reassurance to health professionals who 275 assess and advise parents of children with food allergy. 


\section{References}

1. Venter C, Pereira B, Voigt K, Grundy J, Clayton CB, Higgins B, et al. Prevalence and cumulative incidence of food hypersensitivity in the first 3 years of life. Allergy. 2008;63(7):354-9.

2. Venter C, Pereira B, Grundy J, Clayton CB, Roberts G, Higgins B, et al. Incidence of parentally reported and clinically diagnosed food hypersensitivity in the first year of life. J Allergy Clin Immunol. 2006;117:1118-24.

3. Eggesbø M, Botten $\mathrm{G}$, Stigum $\mathrm{H}$. Restricted diets in children with reactions to milk and egg perceived by their parents. J Pediatr. 2001;139:583-7.

4. Sinagra JL, Bordignon V, Ferraro C, Cristaudo a., Di Rocco M, Amorosi B, et al. Unnecessary milk elimination diets in children with atopic dermatitis. Pediatr Dermatol. 2007;24(1):1-6.

5. Dovey TM, Staples P a., Gibson EL, Halford JCG. Food neophobia and "picky/fussy" eating in children: A review. Appetite. 2008;50:181-93.

6. Wright CM, Parkinson KN, Shipton D, Drewett RF. How do toddler eating problems relate to their eating behavior, food preferences, and growth? Pediatrics. 2007;120:e1069-75.

7. Carruth BR, Ziegler PJ, Gordon A, Barr SI. Prevalence of picky eaters among infants and toddlers and their caregivers' decisions about offering a new food. J Am Diet Assoc. 2004;104:57-64.

8. Carruth BR, Skinner JD. Feeding behaviors and other motor development in healthy children (2-24 months). J Am Coll Nutr. 2002;21:88-96.

9. Crist W, Napier-Phillips A. Mealtime Behaviors of Young Children: A Comparison of Normative and Clinical Data. J Dev Behav Pediatr. 2001;22(5):279-86.

10. Lewinsohn PM, Holm-Denoma JM, Gau JM, Joiner TE, Striegel-Moore R, Bear $\mathrm{P}$, et al. Problematic eating and feeding behaviors of 36-month-old children. Int J Eat Disord. 2005;38:208-19.

11. Levy Y, Levy A, Zangen T, Kornfeld L, Dalal I, Samuel E, et al. Diagnostic clues for identification of nonorganic vs organic causes of food refusal and poor feeding. J Pediatr Gastroenterol Nutr. 2009;48:355-62.

12. Haas AM. Feeding disorders in food allergic children. Current Allergy and Asthma Reports. 2010. p. 258-64.

13. Meyer R, Rommel N, Van Oudenhove L, Fleming C, Dziubak R, Shah N. Feeding difficulties in children with food protein induced gastrointestinal allergies. J Gastroenterol Hepatol [Internet]. 2014;1-21. Available from: http://www.ncbi.nlm.nih.gov/pubmed/24720353 
14. Mukkada VA, Haas A, Maune NC, Capocelli KE, Henry M, Gilman N, et al. Feeding dysfunction in children with eosinophilic gastrointestinal diseases. Pediatrics. 2010;126:e672-7.

15. Carruth BR, Skinner J, Houck K, Moran J, Coletta F, Ott D. The phenomenon of "picky eater": a behavioral marker in eating patterns of toddlers. J Am Coll Nutr. 1998;17(2):180-6.

16. Ramsay M, Martel C, Porporino M, Zygmuntowicz C. The Montreal children's hospital feeding scale: A brief bilingual screening tool for identifying feeding problems. Paediatr Child Health (Oxford). 2011;16(3):147-51.

17. Wu YP, Franciosi JP, Rothenberg ME, Hommel KA. Behavioral feeding problems and parenting stress in eosinophilic gastrointestinal disorders in children. Pediatr Allergy Immunol. 2012;23:730-5.

18. Pentiuk SP, Miller CK, Kaul A. Eosinophilic esophagitis in infants and toddlers. Dysphagia [Internet]. 2007;22:44-8. Available from: http://www.ncbi.nlm.nih.gov/entrez/query.fcgi?cmd=Retrieve\&db=PubMed\&do pt=Citation\&list_uids $=17024545$

19. Davis AM, Bruce AS, Khasawneh R, Shultz T, Fox C, Dunn W. outpatient feeding clinic: A retrospective chart review. J Pediatr Gastroenterol Nutr. 2013;56(2):156-60.

20. Powers SW, Mitchell MJ, Patton SR, Byars KC, Jelalian E, Mulvihill MM, et al. Mealtime behaviors in families of infants and toddlers with cystic fibrosis. J Cyst Fibros. 2005;4(3):175-82.

21. Burklow K a, McGrath AM, Valerius KS, Rudolph C. Relationship between feeding difficulties, medical complexity, and gestational age. Nutr Clin Pract. 2002;17(6):373-8.

22. Northstone $\mathrm{K}$, Emmett $\mathrm{P}$, Nethersole $\mathrm{F}$. The effect of age of introduction to lumpy solids on foods eaten and reported feeding difficulties at 6 and 15 months. J Hum Nutr Diet. 2001;14:43-54.

23. Coulthard H, Harris G, Emmett P. Delayed introduction of lumpy foods to children during the complementary feeding period affects child's food acceptance and feeding at 7 years of age. Matern Child Nutr. 2009;5:75-85.

24. Blossfeld I, Collins a., Kiely M, Delahunty C. Texture preferences of 12-monthold infants and the role of early experiences. Food Qual Prefer. 2007;18:396404.

25. Tharner A, Jansen PW, Kiefte-de Jong JC, Moll H a., Hofman A, Jaddoe VWV, et al. Bidirectional Associations between Fussy Eating and Functional Constipation in Preschool Children. J Pediatr [Internet]. Elsevier Inc; 2015;166(1):91-6.e1. Available from: http://linkinghub.elsevier.com/retrieve/pii/S0022347614008798 
26. Bandini LG, Anderson SE, Curtin C, Cermak S, Evans EW, Scampini R, et al. Food selectivity in children with autism spectrum disorders and typically developing children. J Pediatr. 2010;157(2):259-64.

27. De Lauzon-Guillain B, Oliveira A, Charles MA, Grammatikaki E, Jones L, Rigal N, et al. A Review of Methods to Assess Parental Feeding Practices and Preschool Children's Eating Behavior: The Need for Further Development of Tools. J Acad Nutr Diet. 2012;112.

28. Bryant-Waugh R, Markham L, Kreipe RE, Walsh BT. Feeding and eating disorders in childhood. Int J Eat Disord. 2010;43(2):98-111.

29. Organisation WH. International Statistical Classification of Diseases and Related Health Problems 10th Revision (ICD-10)-2015. 10th revis. World Health Organisation; 2015.

30. Association AP. Diagnostic and Statistical Manual of Mental Disorders Fourth Edition. Fourth Edi. Washington DC: American Psychiatric Association;

31. Levine A, Bachar L, Tsangen Z, Mizrachi A, Levy A, Dalal I, et al. Screening criteria for diagnosis of infantile feeding disorders as a cause of poor feeding or food refusal. J Pediatr Gastroenterol Nutr. 2011;52(5):563-8.

32. Mitchell GL, Farrow C, Haycraft E, Meyer C. Parental influences on children's eating behaviour and characteristics of successful parent-focussed interventions. Appetite [Internet]. Elsevier Ltd; 2013;60(1):85-94. Available from: http://dx.doi.org/10.1016/j.appet.2012.09.014 